\title{
Lie reductions for the Euler-Bernoulli model of flexible beam robots
}

\author{
Mehdi Nadjafikhah ${ }^{1}$, Leila Hamedi Mobarra ${ }^{2}$ \\ ${ }^{1}$ (Mathematics Department/ Iran University of Science and Technology, Iran) \\ ${ }_{2}^{2}$ Science Department/ Payamnoor University, Iran)
}

\begin{abstract}
The Euler-Bernoulli model of flexible beam robots is a four-dimensional under-determined partial differential equation, related to define the unique link's deflection of this type of robots. In this paper, Lie point symmetries of the equation have been investigated by a Lie group method. Then, reductions of the Euler-Bernoulli equation to ordinary equations have been obtained by repeat the method. Two of the reduced equations are of the first order.
\end{abstract}

Keywords: Euler-Bernoulli model of flexible beam robots, Symmetry algebra, Lie point symmetry, Optimal system of sub-algebras, Lie reduction of equation

\subsection{Classical Lie point symmetries of PDEs}

\section{Introduction}

Sophus Lie and et al innovated the methods for finding Lie point symmetries and reductions of differential equations in the 19th century. They sought the linearized symmetry condition for infinitesimal transformations. The linearized condition for a classical infinitesimal one-dimensional Lie point symmetry $X$ corresponded to a k-dimensional PDE, $\Delta=0$, is given by (ref to [1]):

$$
\left.p r^{(k)} X(\Delta)\right|_{\Delta=0}=0 \text {. }
$$

Calculating invariants for each of one-parameter infinitesimal Lie point symmetries lead to find reductions of the equation. In this work, for the Euler-Bernoulli PDE of the fourth order, with two independent variables and two dependent variables, Lie reductions are repeatedly obtained. The reduced equations involve one independent variable and two dependent variables. Two of the reduced equations are the first order ODEs.

\subsection{Flexible beam robots}

In two recent decades, flexible robots have been paid much attention in mechanics and robotic. In particular, a robot with three joints and two links that one of the two links is elastic and the deformation of (elastic) link 2 can happen along a direction perpendicular to the longitudinal axis of link 2, was exhaustively studied by Wenhuan Yu. According to [2], Yu acquired a mathematical model for this class of flexible robots in 1994. Indeed, he with citing to $[3,4,5,6,7,8,9]$, at first, produced a system which is composed of two elastic partial differential equations and three nonlinear integrodifferential equations for a general robot of that class. Thereupon, he exhibited three special cases of that mathematical model. Here, we focus on one of those specials which is concerned with flexible beam robots. A robot with an unitary flexible link is named a flexible beam robot.

\subsection{Euler-Bernoulli model}

Consider a motor driven beam robot (refer to [10]) with a joint and an elastic link that can deform along a direction perpendicular to its longitudinal axis. In the other, assume that the link is located in the horizontal plane. Likewise, let an orthogonal frame $\{i, j\}$ at the joint point in the plane, such that the axis $i$ is tangent to the link at the point and the axis $j$ is the direction of deformation. Now, install a DC motor at the joint which deflects the link around the axis i. Besides, suppose that $t$ is the time variable, similarly, $x$ is the distance between an arbitrary point on the link and the joint. Furthermore, $R(x, t)$ is the vector from the joint to the corresponding point to $x$, at time $t$. Afterward, other necessary information of the robot are presented in the Table 1:

Table 1: The information of the beam robot

\begin{tabular}{|c|c|}
\hline$v(x, t)$ & bending of point $x$ at time $t$, along the direction $j$ \\
\hline$\rho(x)$ & linear density of the link at $x$ \\
\hline$E(x)$ & the Yong's modulus of elasticity \\
\hline$J(x)$ & inertia moment of the link, with respect to the direction $i$ at $x$ \\
\hline$p(x)=E(x) J(x)$ & bending stiffness of the link at $x$ \\
\hline
\end{tabular}


It should be noted that $R(x, t)=x i+v(x, t) j$. On the other hand, by very detailed calculation, the mathematical model of the flexible beam robot has been obtained as (refer to [11, 12])

$$
\begin{aligned}
& \Delta(t, x, v, p)=\rho(x) v_{t t}+p^{\prime \prime} v_{x x}+2 p^{\prime} v_{x x x}+p v_{x x x x}, \\
& \left(\text { where } p^{\prime}=p_{x}, p^{\prime \prime}=p_{x x}\right) .
\end{aligned}
$$

On the basis of [10], this partial differential equation is an Euler- Bernoulli equation. It is the same Euler-Bernoulli model of flexible beam robots. In this research, a Lie point symmetry group for this PDE is represented. Then, sub-algebras are classified. Finally, the reductions of the equation to ODEs by optimal sub-algebras, are found.

\subsection{The Lie point symmetry algebra}

\section{The symmetry group}

Take infinitesimal generators of the Lie algebra of a Lie point symmetry group of (2), in the form

$$
X=\xi_{1} \partial_{t}+\xi_{2} \partial_{x}+\phi_{1} \partial_{v}+\phi_{2} \partial_{p}
$$

where $\xi_{1}, \xi_{2}, \phi_{1}, \phi_{2}$ are $C^{\infty}$ functions of $t, x, v, p$.

Applying the classical linearized symmetry condition ( 1) results the following linearly independent vector fields

$$
\begin{aligned}
& u_{1}=-\frac{1}{2} t \partial_{t}-v \partial_{v}+p \partial_{p}, \\
& u_{2}=x \partial_{v}, \\
& u_{3}=\partial_{v}, \\
& u_{4}=t x \partial_{v}, \\
& u_{5}=t \partial_{v} . \\
& u_{6}=\partial_{t} .
\end{aligned}
$$

Their fourth prolongations are according to

$$
\begin{aligned}
& p r^{(4)} u_{1}=-\frac{1}{2} t \partial_{t}-v \partial_{v}+p \partial_{p}-\frac{1}{2} v_{t} \partial_{v_{t}}-v_{x} \partial_{v_{x}}+p_{x} \partial_{p_{x}}-\frac{1}{2} v_{t x} \partial_{v_{t x}} \\
& -v_{x x} \partial_{v_{x x}}+p_{x x} \partial_{p_{x x}}+\frac{1}{2} v_{t t t} \partial_{v_{t t t}}-\frac{1}{2} v_{t x x} \partial_{v_{t x x}}-v_{x x x} \partial_{v_{x x x}} \\
& +p_{x x x} \partial_{p_{x x x}}+v_{t t t t} \partial_{v_{t t t t}}+\frac{1}{2} v_{t t t x} \partial_{v_{t t t x}}-\frac{1}{2} v_{t x x x} \partial_{v_{t x x x}} \\
& -v_{x x x x} \partial_{v_{x x x x}}+p_{x x x x} \partial_{p_{x x x x}}, \\
& p r^{(4)} u_{2}=x \partial_{v}+\partial_{v_{x}}, \quad(5) \\
& p r^{(4)} u_{3}=\partial_{v} \\
& p r^{(4)} u_{4}=t x \partial_{v}+x \partial_{t}+t \partial_{v_{x}}+\partial_{v_{t x}} \\
& p r^{(4)} u_{5}=t \partial_{v}+\partial_{v_{t}} \\
& p r^{(4)} u_{6}=\partial_{t} .
\end{aligned}
$$

Hence

$$
\left.p r^{(4)} u_{i}(\Delta)\right|_{\Delta=0}=0 . \quad(i=1,2,3,4,5,6)
$$

Thus $\mathrm{g}=\left\langle u_{1}, u_{2}, u_{3}, u_{4}, u_{5}, u_{6}\right\rangle$ is a Lie algebra corresponding to a symmetry group of the EulerBernoulli equation. It is known as the symmetry algebra.

\subsection{The Lie point symmetry group}

Let $G$ be the Lie point group related to the symmetry algebra $\mathrm{g}$. $G$ is a Lie point symmetry group for 
the Euler- Bernoulli equation which is produced by one-parameter transformations $\exp \left(\varepsilon u_{i}\right)$ $(i=1,2,3,4,5,6), u_{i} \in \mathrm{g}, \varepsilon \in \mathrm{R}$. It is obvious that each of these transformations creates a one-parameter subgroup of $G$. Because of this equation involves two independent variables and two dependent variables, it can be accounted as a total space $E ; \mathrm{P}^{2+2}$ with coordinates $t, x, v, p$. Then, the Lie point transformations are via

$$
\begin{aligned}
& \bar{t}=t+\varepsilon \xi_{1}(t, x, v, p)+\mathrm{O}\left(\varepsilon^{2}\right), \\
& \bar{x}=x+\varepsilon \xi_{2}(t, x, v, p)+\mathrm{O}\left(\varepsilon^{2}\right), \\
& \bar{v}=v+c \phi_{1}(t, x, v, p)+\mathrm{O}\left(\varepsilon^{2}\right), \\
& \bar{p}=p+c \phi_{2}(t, x, v, p)+\mathrm{O}\left(\varepsilon^{2}\right),
\end{aligned}
$$

where $\xi_{1}, \xi_{2}, \phi_{1}, \phi_{2}$ are $C^{\infty}$ functions.

Note that for per $w=(t, x, v, p)$ belonging to the total Euclidean space $E$ and $\varepsilon \in \mathrm{R}$, the local flow $\exp \left(\varepsilon u_{i}\right)(w)$ is defined by

$$
\partial_{\varepsilon} \exp \left(\varepsilon u_{i}\right)(w)=\left.u_{i}\right|_{\exp \left(\varepsilon u_{i}\right)(w)},\left.\quad \exp \left(\varepsilon u_{i}\right)(w)\right|_{\varepsilon=0}=w . \quad\left(\text { where } \partial_{\varepsilon}=\frac{d}{d \varepsilon}\right)
$$

Therefore, the operation of one-parameter Lie point transformations $\exp \left(\varepsilon u_{i}\right): E \rightarrow E$, namely is computed as

$$
\exp \left(\varepsilon u_{i}\right):(t, x, v, p) \mapsto(\bar{t}, \bar{x}, \bar{v}, \bar{p}), \quad(i=1,2,3,4,5,6, \quad \varepsilon \in \mathrm{P})
$$

Table 2: One-parameter subgroups actions

\begin{tabular}{|c||c|c|c|c|}
\hline & $\bar{t}$ & $\bar{x}$ & $\bar{v}$ & $\bar{p}$ \\
\hline $\exp \left(\varepsilon u_{1}\right)$ & $e^{-\frac{1}{2} \varepsilon} t$ & $x$ & $e^{-\varepsilon} v$ & $e^{\varepsilon} p$ \\
\hline $\exp \left(\varepsilon u_{2}\right)$ & $t$ & $x$ & $v+\varepsilon x$ & $p$ \\
\hline $\exp \left(\varepsilon u_{3}\right)$ & $t$ & $x$ & $v+\varepsilon$ & $p$ \\
\hline $\exp \left(\varepsilon u_{4}\right)$ & $t$ & $x$ & $v+\varepsilon t x$ & $p$ \\
\hline $\exp \left(\varepsilon u_{5}\right)$ & $t$ & $x$ & $v+\varepsilon t$ & $p$ \\
\hline $\exp \left(\varepsilon u_{6}\right)$ & $t+\varepsilon$ & $x$ & $v$ & $p$ \\
\hline
\end{tabular}

It is worth mentioning that for each $g$ belonging to $G$, there are vectors $u_{1}, \cdots, u_{k}$ $(k=1,2,3,4,5,6)$ of $g$, such that $g=\exp u_{1} \circ \cdots \circ \exp u_{k}$. Afterward, since the action of $G$ on $E$ is obtained by combination of the one-parameter subgroups actions, the use of the Table 2 results:

Theorem 2.1 If $(v, p)=(F(t, x), G(t, x))$ is a solution of the Euler-Bernoulli equation then so are

$$
\begin{aligned}
& (v, p)=\left(e^{-\varepsilon} F\left(e^{-\frac{1}{2} \varepsilon} t, x\right), e^{\varepsilon} G\left(e^{-\frac{1}{2} \varepsilon} t, x\right)\right), \\
& (v, p)=(F(t, x)+\varepsilon x, G(t, x)), \\
& (v, p)=(F(t, x)+\varepsilon, G(t, x)), \\
& (v, p)=(F(t, x)+\varepsilon t x, G(t, x)), \\
& (v, p)=(F(t, x)+\varepsilon t, G(t, x)), \\
& (v, p)=(F(t+\varepsilon, x), G(t+\varepsilon, x)),
\end{aligned}
$$

where $\varepsilon$ is an arbitrary parameter.

Account general vector $V$ as 


$$
V=\alpha_{1} u_{1}+\cdots+\alpha_{6} u_{6} . \quad\left(\alpha_{1}, \cdots, \alpha_{6} \in \mathrm{R}\right)
$$

Computating $\exp (\varepsilon V)$ deduces:

Corollary 2.2 If $v=F(t, x), \quad p=G(t, x)$ is a solution of the Euler-Bernoulli equation then so is

$$
\begin{aligned}
& v=F\left(t+c_{1} e^{-\frac{1}{2} c_{2} \varepsilon}+c_{3}, x\right)+c_{4} t+c_{5} x+c_{6} t x+ \\
& \left(c_{7}+c_{8} x\right) e^{-\frac{1}{2} c_{2} \varepsilon}+c_{9} e^{-c_{2} \varepsilon}, \\
& p=e^{c_{2} \varepsilon} G\left(t+c_{1} e^{-\frac{1}{2} c_{2} \varepsilon}+c_{3}, x\right),
\end{aligned}
$$

where $\varepsilon, c_{i}(i=1,2, \cdots, 9)$ are arbitrary numbers.

\subsection{Lie algebra structure}

\section{Structure of the symmetry algebra} series is

This Lie algebra is non-nilpotent and solvable; because it has no upper central series, and its lower central

$$
\mathrm{g} \supset\left\langle 2 u_{2}, 2 u_{3}, u_{4}, u_{5}, u_{6}\right\rangle \supset\left\langle 4 u_{2}, 4 u_{3}, u_{4}, u_{5}, u_{6}\right\rangle \text {. }
$$

Also, $g$ is not semi simple because its killing form is generated as the following

$$
\left(\begin{array}{cccccc}
\frac{11}{4} & 0 & 0 & 0 & 0 & 0 \\
0 & 0 & 0 & 0 & 0 & 0 \\
0 & 0 & 0 & 0 & 0 & 0 \\
0 & 0 & 0 & 0 & 0 & 0 \\
0 & 0 & 0 & 0 & 0 & 0 \\
0 & 0 & 0 & 0 & 0 & 0
\end{array}\right)
$$

On the other, based on $\left[u_{i}, u_{j}\right]=u_{i} u_{j}-u_{j} u_{i}(i, j=1,2,3,4,5,6)$, the commutator table of Lie algebra $g$ is constructed as the next table:

Table 3: Commutation relations

\begin{tabular}{|c||c|c|c|c|c|c|}
\hline & $u_{1}$ & $u_{2}$ & $u_{3}$ & $u_{4}$ & $u_{5}$ & $u_{6}$ \\
\hline \hline$u_{1}$ & 0 & $u_{2}$ & $u_{3}$ & $\frac{1}{2} u_{4}$ & $\frac{1}{2} u_{5}$ & $\frac{1}{2} u_{6}$ \\
\hline$u_{2}$ & $-u_{2}$ & 0 & 0 & 0 & 0 & 0 \\
\hline$u_{3}$ & $-u_{3}$ & 0 & 0 & 0 & 0 & 0 \\
\hline$u_{4}$ & $-\frac{1}{2} u_{4}$ & 0 & 0 & 0 & 0 & $-u_{2}$ \\
\hline$u_{5}$ & $-\frac{1}{2} u_{5}$ & 0 & 0 & 0 & 0 & $-u_{3}$ \\
\hline$u_{6}$ & $-\frac{1}{2} u_{6}$ & 0 & 0 & $u_{2}$ & $u_{3}$ & 0 \\
\hline
\end{tabular}

Therefore, $\mathbf{g}$ is not Abelian. Nevertheless, its Abelian sub-algebras are as: 


$$
\begin{aligned}
& \left\langle c_{1} u_{1}+c_{2} u_{2}+c_{3} u_{3}+c_{4} u_{4}+c_{5} u_{5}+c_{6} u_{6}\right\rangle, \\
& \left\langle u_{2}, c_{3} u_{3}+c_{4} u_{4}+c_{5} u_{5}+c_{6} u_{6}\right\rangle,\left\langle u_{3}, c_{4} u_{4}+c_{5} u_{5}+c_{6} u_{6}\right\rangle,\left\langle u_{4}, u_{5}\right\rangle, \\
& \left\langle u_{2}, u_{3}, c_{4} u_{4}+c_{5} u_{5}+c_{6} u_{6}\right\rangle,\left\langle u_{3}, u_{4}, u_{5}\right\rangle \\
& \left\langle u_{2}, u_{3}, u_{4}, u_{5}\right\rangle
\end{aligned}
$$

(where $c_{1}, c_{2}, c_{3}, c_{4}, c_{5}, c_{6}$ are arbitrary constants)

Besides, the center of $g$ is empty. The optimal systems of sub-algebras follow as the next subsection.

\subsection{Classification of sub-algebras}

Using the Table 3 for the Lie series $\operatorname{A} d\left(\exp \left(\varepsilon u_{i}\right)\right) u_{j}=u_{i}-\varepsilon\left[u_{i}, u_{j}\right]+\frac{\varepsilon^{2}}{2}\left[u_{i},\left[u_{i}, u_{j}\right]\right]-\cdots$, generates the subsequent table:

Table 4: Adjoint actions

\begin{tabular}{|c|c|c|c|c|c|c|}
\hline Ad & $u_{1}$ & $u_{2}$ & $u_{3}$ & $u_{4}$ & $u_{5}$ & $u_{6}$ \\
\hline$u_{1}$ & $u_{1}$ & $e^{\varepsilon} u_{2}$ & $e^{\varepsilon} u_{3}$ & $e^{\frac{1}{2} \varepsilon} u_{4}$ & $e^{\frac{1}{2} \varepsilon} u_{5}$ & $e^{\frac{1}{2} \varepsilon} u_{6}$ \\
\hline$u_{2}$ & $u_{1}$ & $u_{2}-\varepsilon u_{1}$ & $u_{3}$ & $u_{4}$ & $u_{5}$ & $u_{6}$ \\
\hline$u_{3}$ & $u_{1}$ & $u_{2}$ & $u_{3}-\varepsilon u_{1}$ & $u_{4}$ & $u_{5}$ & $u_{6}$ \\
\hline$u_{4}$ & $u_{1}$ & $u_{2}-\varepsilon u_{6}$ & $u_{3}$ & $u_{4}-\frac{1}{2} \varepsilon u_{1}$ & $u_{5}$ & $u_{6}$ \\
\hline$u_{5}$ & $u_{1}$ & $u_{2}$ & $u_{3}-\varepsilon u_{6}$ & $u_{4}$ & $u_{5}-\frac{1}{2} \varepsilon u_{1}$ & $u_{6}$ \\
\hline$u_{6}$ & $u_{1}$ & $u_{2}+\varepsilon u_{4}$ & $u_{3}+\varepsilon u_{5}$ & $u_{4}$ & $u_{5}$ & $u_{6}-\frac{1}{2} \varepsilon u_{1}$ \\
\hline
\end{tabular}

(with the $(i, j)$-th entry indicating $\left.\mathrm{A} d\left(\exp \left(\varepsilon u_{i}\right)\right) u_{j}(i, j=1,2,3,4,5,6)\right)$

Hence, for (10), the matrix of $\mathrm{A} d\left(\exp \left(\varepsilon_{1} u_{1}\right)\right) \circ \cdots \circ \mathrm{A} d\left(\exp \left(\varepsilon_{6} u_{6}\right)\right) V$ is determined as

$$
\left(\begin{array}{cccccc}
1 & 0 & 0 & 0 & 0 & 0 \\
-e^{\varepsilon_{1}} \varepsilon_{2}+\frac{1}{2} e^{\varepsilon_{1}} \varepsilon_{4} \varepsilon_{6} & e^{\varepsilon_{1}} & 0 & e^{\varepsilon_{1}} \varepsilon_{6} & 0 & -e^{\varepsilon_{1}} \varepsilon_{4} \\
-e^{\varepsilon_{1}} \varepsilon_{3}+\frac{1}{2} e^{\varepsilon_{1}} \varepsilon_{5} \varepsilon_{6} & 0 & e^{\varepsilon_{1}} & 0 & e^{\varepsilon_{1}} \varepsilon_{6} & -e^{\varepsilon_{1}} \varepsilon_{5} \\
-\frac{1}{2} e^{\frac{1}{2} \varepsilon_{1}} \varepsilon_{4} & 0 & 0 & e^{\frac{1}{2} \varepsilon_{1}} & 0 & 0 \\
-\frac{1}{2} e^{\frac{1}{2} \varepsilon_{1}} \varepsilon_{5} & 0 & 0 & 0 & e^{\frac{1}{2} \varepsilon_{1}} & 0 \\
-\frac{1}{2} e^{\frac{1}{2} \varepsilon_{1}} \varepsilon_{6} & 0 & 0 & 0 & 0 & e^{\frac{1}{2} \varepsilon_{1}}
\end{array}\right),
$$

which leads us to the next theorem proof. 
Theorem 3.1 A one dimensional optimal system of symmetric sub-algebras of the Euler-Bernoulli equation is conformity with

$$
\left\langle u_{1}+\sum_{i=2}^{6} c_{i} u_{i}\right\rangle, \quad\left\langle u_{4}+a u_{5}+b u_{6}\right\rangle,
$$

where $c_{i}(i=1,2,3,4,5,6)$ and $a, b$ are arbitrary and nonzero constants, respectively.

For every $i(1 \square i \square 5)$, if $V_{\alpha}(\square \alpha \square i)$ are generators of a definite member of $i$-dimensional optimal system, then providing this vectors with together an appropriate $V$ (refer to (10)) construct a Lie algebra, necessitates that $\left\langle V, V_{1}, \cdots V_{i}\right\rangle$ is a member of $(i+1)$-dimensional optimal system. In general, it can be obtained:

Table 5: Optimal systems of sub-algebras

\begin{tabular}{|c||c|}
\hline dimension & Sub-algebras \\
\hline \hline 1 & $\left\langle u_{1}+\sum_{i=2}^{6} c_{i} u_{i}\right\rangle,\left\langle u_{4}+a u_{5}+b u_{6}\right\rangle$ \\
\hline 2 & $\left\langle u_{1}+\sum_{i=2}^{6} c_{i} u_{i}, u_{2}+c_{3} u_{3}\right\rangle,\left\langle u_{1}+c_{2} u_{2}+c_{3} u_{3}, u_{4}+a u_{5}+b u_{6}\right\rangle$ \\
\hline 3 & $\left\langle u_{1}+\sum_{i=2}^{6} c_{i} u_{i}, u_{2}+c_{3} u_{3}, u_{3}\right\rangle,\left\langle u_{1}+c_{2} u_{2}+c_{3} u_{3}, u_{4}+a u_{5}+b u_{6}, u_{2}+c_{3} u_{3}\right\rangle$ \\
\hline 4 & $\left\langle u_{1}+\sum_{i=2}^{6} c_{i} u_{i}, u_{2}+c_{3} u_{3}, u_{3}, u_{4}\right\rangle,\left\langle u_{1}+\sum_{i=2}^{6} c_{i} u_{i}, u_{2}+c_{3} u_{3}, u_{3}, u_{5}\right\rangle$, \\
& $\left\langle u_{1}+\sum_{i=2}^{6} c_{i} u_{i}, u_{2}+c_{3} u_{3}, u_{3}, u_{6}\right\rangle$ \\
\hline 5 & $\left\langle u_{1}+\sum_{i=2}^{6} c_{i} u_{i}, u_{2}+c_{3} u_{3}, u_{3}, u_{4}, u_{5}\right\rangle,\left\langle u_{1}+\sum_{i=2}^{6} c_{i} u_{i}, u_{2}+c_{3} u_{3}, u_{3}, u_{4}, u_{6}\right\rangle$ \\
\hline 6 & 9 \\
\hline
\end{tabular}

(where $c_{i}(i=1,2,3,4,5,6)$ and $a, b$ are arbitrary and nonzero constants, respectively.)

\section{Lie reductions of the equation by the optimal systems of sub-algebras}

Constructing and solving the characteristic equations for generators of the optimal symmetric sub-algebras produce differential invariants of these sub-algebras as the table:

Table 6: Invariants of up to three-dimensional optimal systems

\begin{tabular}{|c|c|}
\hline sub-algebras & differential invariants up to the first order \\
\hline$\left\langle u_{1}+\sum_{i=2}^{6} c_{i} u_{i}\right\rangle$ & $x, \frac{v-2 t\left(c_{5}+c_{4} x\right)+x\left(2 c_{4} c_{6}-c_{2}\right)+2 c_{5} c_{6}-c_{3}}{\left(2 c_{6}-t\right)^{2}}, p\left(2 c_{6}-t\right)^{2}$, \\
$v_{t}, v_{x}, p^{\prime}$
\end{tabular}

Each of the first two rows in the Table 6 includes three ordinary differential invariants. More generally, apart from the penultimate row, for each of the other rows can be defined three ordinary invariants. Ordinary invariants are applied to formulate relations that substituting them into the Euler- Bernoulli equation reduces the number of its independent variables. This fact is the first stage of reductions. Also, repeating the mentioned manner for the reduced equations, constructs the later stages of reductions (refer to [12]). As is following: 
i) The reduction by $\left\langle u_{1}+\sum_{i=2}^{6} c_{i} u_{i}\right\rangle$ is done by the formula

$$
\begin{gathered}
v(t, x)=\left(t-2 c_{6}\right)^{2} w(z)+c_{2} z-2 c_{5} c_{6}+2 c_{4} t z+2 c_{5} t+c_{3}, \\
p(x)=\frac{s(z)}{\left(t-2 c_{6}\right)^{2}}, \quad x=z, \quad(17)
\end{gathered}
$$

and the reduced equation is

$$
2 \rho(z) w+s_{z z} w_{z z}+2 s_{z} w_{z z z}+s w_{z z z}=0 .
$$

The equation (18), does not have any symmetry.

ii) The reduction by $\left\langle u_{4}+a u_{5}+b u_{6}\right\rangle$ is done by the formula

$$
v(t, x)=w(z)+\frac{1}{2 b}(z+a) t^{2}, \quad p(x)=s(z), \quad x=z,
$$

and the reduced equation is

$$
\frac{1}{b}(z+a) \rho(z)+s_{z z} w_{z z}+2 s_{z} w_{z z z}+s w_{z z z z}=0 .
$$

( where $a, b$ are nonzero constants. )

Eq.(20) has a symmetry group with infinitesimals $\alpha_{1}=-w \partial_{w}+s \partial_{s}, \alpha_{2}=z \partial_{w}, \alpha_{3}=\partial_{w}$. $\left\langle\alpha_{1}\right\rangle$ doesn't reduce (18).

- The reduction by $\left\langle\alpha_{2}\right\rangle$ and $\left\langle\alpha_{3}\right\rangle$ is given by

$$
s(w)=V(f), w(z)=\int h(f) d f, z=f,
$$

and the reduced equation is

$$
\frac{1}{b}(f+a) \rho(z)+V_{f f} h_{f}+2 V_{f} h_{f f}+V h_{f f f}=0 .
$$

Eq.(22) has a symmetry group with infinitesimal $\beta_{1}=\partial_{h}$.

- The reduction by $\left\langle\beta_{1}\right\rangle$ is given by

$$
V(f)=S(F), h(f)=\int W(F) d F, f=F,
$$

and the reduced equation is

$$
\frac{1}{b}(F+a) \rho(F)+W S_{F F}+2 S_{F} W_{F}=0 .
$$

Eq.(24) has a symmetry group with infinitesimals $\eta_{1}=-s \partial_{s}+W \partial_{s}, \eta_{2}=\partial_{s}$. $\left\langle\eta_{1}\right\rangle$ doesn't reduce $(24)$.

- The reduction by $\left\langle\eta_{2}\right\rangle$ is given by

$$
W(F)=\mathscr{W}(\mathscr{F}), S(F)=\int \mathscr{W}(\mathscr{F}) d \mathscr{F}, F=\mathscr{F},
$$

and the reduced equation is

$$
\frac{1}{b}(\mathscr{F}+a) \rho(\mathscr{F})+\mathscr{W} S_{\mathscr{F}}+2 S_{\mathscr{F}} \mathscr{W}_{\mathscr{F}}=0 .
$$

iii) The reduction by $\left\langle u_{1}+\sum_{i=2}^{6} c_{i} t_{i}, t_{2}+c_{3} u_{3}\right\rangle$ is done by the formula

$$
v(t, x)=\int w(z) d t, \quad p(x)=\frac{s(z)}{\left(2 c_{6}-2\right)^{2}}, \quad x=z,
$$

and the reduced equation is

$$
s_{z z} w_{z z}+2 s_{z} w_{z z z}+s w_{z z z z}=0 .
$$

Eq.(28) has a symmetry group with infinitesimals $\gamma_{1}=\frac{1}{4} z \partial_{z}+s \partial_{s}, \gamma_{2}=\frac{1}{4} z \partial_{z}+w \partial_{w}, \gamma_{3}=\partial_{w}, \gamma_{4}=$ $z \partial_{w}, \gamma_{5}=\partial_{z .}\left\langle\gamma_{1}\right\rangle$ and $\left\langle\gamma_{5}\right\rangle$ don't reduce (28). 
- The reduction by $\left\langle\gamma_{2}\right\rangle,\left\langle\gamma_{3}\right\rangle$ and $\left\langle\gamma_{4}\right\rangle$ is given by

$$
s(w)=V(f), w(z)=\int h(f) d f, z=f,
$$

and the reduced equation is

$$
V_{f f} h_{f}+2 V_{f} h_{f f}+V h_{f f f}=0 .
$$

Eq.(30) has a symmetry group with infinitesimals $\delta_{1}=\frac{1}{3} f \partial_{f}+V \partial_{V}, \delta_{2}=\partial_{f}$.

$\left\langle\delta_{2}\right\rangle$ doesn't reduce $(30)$.

- The reduction by $\left\langle\delta_{1}\right\rangle$ is given by

$$
V(f)=S(F), h(f)=\int W(F) d F, f=F,
$$

and the reduced equation is

$$
W S_{F F}+2 S_{F} W_{F}=0 .
$$

Eq.(32) has a symmetry group with infinitesimals $\zeta_{1}=\frac{1}{2} F \partial_{F}+W \partial_{W}, \zeta_{2}=\frac{1}{2} F \partial_{F}+S \partial_{S}, \zeta_{3}=$ $\partial_{S}, \zeta_{4}=\partial_{F} \cdot\left\langle\zeta_{1}\right\rangle$ and $\left\langle\zeta_{4}\right\rangle$ doesn't reduce (32).

- The reduction by $\left\langle\zeta_{2}\right\rangle,\left\langle\zeta_{3}\right\rangle$ is given by

$$
W(F)=\mathscr{W}(\mathscr{F}), S(F)=\int \mathscr{W}(\mathscr{F}) d \mathscr{F}, F=\mathscr{F},
$$

and the reduced equation is

$$
\mathscr{W} S_{\mathscr{F}}+2 S_{\mathscr{F}} \mathscr{W}_{\mathscr{F}}=0 .
$$

iv) The reduction by $\left\langle u_{1}+\sum_{i=2}^{6} c_{i} u_{i}, t t_{2}+c_{3} u_{3}, u_{3}\right\rangle$ is similar to $\mathrm{i}$ item.

v) The reduction by $\left\langle u_{1}+c_{2} u_{2}+c_{3} u_{3}, u_{4}+a u_{5}+b u_{6}, u_{2}+c_{3} u_{3}\right\rangle$ is similar to iii item.

Remark 4.1 The equation (18) not only isn't symmetric but also isn't non-classical symmetric ${ }^{1}$.

\section{Conclusion}

In this article has been given the details of the symmetry analysis of a certain nonlinear partial differential equation arising in the study of elastic beams, known as Euler-Bernoulli beam equation. At first, a symmetry group has been computed using the standard Lie algorithm. At second, in preparation for the determination of symmetry reductions, the sub-algebras of the symmetry algebra have been classified, using standard techniques. Finally, a list of Lie reductions of the equation, contained of two ODEs has been resulted. The results have simplified the Euler-Bernoulli beam model of a flexible beam robot which its unitary flexible link, moves only in the horizontal plane.

\section{References}

[1] G. W. Bluman, A. F. Cheviakov, and S. C.Anco, Applications of Symmetry Methods to Partial Differential Equations,( NewYork: Springer, 2010).

[2] W. Yu, Mathematical modelling for a class of flexible robot, Appl.Math. Modelling. 19, 1995, $537-542$.

[3] H. T. Banks, and et al, Inverse problems in the modelling of vibrations of flexible beams, Distributed Parameter Systems, Proc. 3rd Int. Conf, Vorau, Syria, July 1986. Springer-Verlag, Berlin 1987.

[4] F. Bellezza, and et al, Exact modelling of the flexible slewing link, Proc. IEEE Int. Conf. on Robotics and Automation, 1990, 734-739.

[5] J. Bontsema, R.F. Curtain, and J.M.Schumacher, Robust control of flexible structures: a case study, Automatica. 24, 1988, $177-186$.

[6] R.Cannon, and Jr.E.Schmitz, Initial experiments on the end-point control of flexible one-link robot, Int. J. Robotics. 3, 1984 , 62-75.

[7] R.F. Curtain, and P. Kotelenez, Stochastic models for uncertain flexible systems, Automatica. 23, 1987, 657-661.

\footnotetext{
${ }^{1}$ A vector field $X$ with the characteristic equation $Q$ on the total space related to a $k$ th order PDE as $\Delta$, is an infinitesimal generator for a non- classical symmetry of $\Delta$, if and only if $\left.\operatorname{Pr}^{(k)} X(\Delta)\right|_{\Delta=0}=0$ and $\left.\operatorname{Pr}^{(1)} X(Q)\right|_{Q=0}=0$ (ref to [1]).
} 
[8] X. Jian, Control problems in robots and robotics(in Chinese), Robotics. 12(5), 1990, 1-12 .

[9] Q.Wang, and et al, Kinematics and dynamics in robots, Robotics. 12(1), 1990, 58-64 .

[10] www.wikipedia.org.

[11] M.O. Tokhi, and A.K.M.Azad, Flexible Robot manipulators modelling simulation and control, ( The Institution of Engineering and Technology, 2008).

[12] P.J. Olver, Applications of Lie groups to differential equations, ( NewYork: Springer, 1986). 